\title{
Actualización De Manejo De Especies De Fauna Silvestre En Cautiverio, En La Región Occidental De Panamá.
}

\author{
Up-Date Management Of Species Of The Wild Fauna In Captivity In The Occidental \\ Region Of Panama.
}

\author{
Susan Marín', Karell Flores²; Jorge Gutiérrez³. \\ ${ }^{1}$ Ministerio de Ambiente, Departamento de Biodiversidad y Vida Silvestre. Panamá, \\ https://orcid.org/0000-0002-7993-8696. Correo: smarin@miambiente.gob.pa \\ 2 Instituto Episcopal San Cristobal. Panamá. \\ https://orcid.org/0000-0002-4807-6261.Correo: k08flor 22@hotmail.com \\ 3 Universidad de Panamá, Departamento de Ciencias Ambientales. Panamá. \\ https://orcid.org/0000-0001-7015-3596. Correo: jlgutiz@yahoo.es.
}

\section{Resumen}

Esta investigación se realizó con el objetivo de actualizar la condición de las especies de fauna silvestres manejadas en cautiverio en la región occidental de Panamá, específicamente en las provincias de Chiriquí, Veraguas, Herrera, Los Santos y Coclé; la cual se llevó a cabo desde el mes de abril a diciembre de 2014, y de enero a junio de 2015. Inicialmente se realizó una revisión de la información contenida en expedientes que reposaban en los archivos del Departamento de Biodiversidad y Vida Silvestre del Ministerio de Ambiente; también se solicitó información a los funcionarios de vida silvestre de las Direcciones Regionales correspondientes del Ministerio de Ambiente, y por último se visitaron los sitios que manejaban especies en cautiverio, tomando en cuenta ciertos aspectos en la toma de datos como: estatus de la actividad, manejo de los animales en cautiverio y la condición de los recintos. En el estudio se registraron un total de 111 sitio de conservación ex situ; de los cuales 102 correspondieron a la actividad de zoocriaderos, 7 a colecciones privadas y 2 a zoológicos; de estos sitios, el estatus de condición ante el ministerio de ambiente correspondió a que 38 se encontraban establecidos legalmente, 38 con estatus ilegal y 35 en trámite de legalización. En cuanto al manejo en cautiverio los resultados arrojaron un total de 100 centros de conservación ex situ que mantenían fauna silvestre en cautiverio en buen estado físico y recintos en buenas condiciones de alojamiento; 109 de los recintos presentaban instalaciones seguras para las especies y 106 de los recintos no contaban con atención veterinaria para los animales de vida silvestre.

Palabras Clave: Especies de fauna silvestre, cautiverio, manejo, conservación, ex situ. 


\begin{abstract}
This investigation was carried-out with the objective to up-date the handling given to wild fauna species held in captivity in the occidental regions of Panama, specifically Chiriqui, Veraguas, Herrera, Los Santos and Cocle, carried-out from April to December 2014, and from January to June 2015. Initially a review of the information was done in files kept at the Regional Direction of Wild Life and Biodiversity of the Ministry of Environment; information was also been requested from the willd life officials of the Regional Direction of the Ministry of Environment and finally sites that handle species in captivity, were visited, considering certain aspects to obtain data, such as: status of the activity, handling of the animals in captivity and condition of the enclosures. In the study, a total of 111 exsitu sites of conservation, were registered, of which 102 corresponded to zoofarms, 7 private collections and 2 zoos. The condition status of these sites before the Ministry of environment showed correspondance of 38 legally stablished, 38 with ilegal status and 35 in legalization process. In terms of handling in captivity, 100 of the conservation exsitu centers that kept wild fauna in captivity, were physically healthy, 109 enclosures were safety for the species, and 106 enclosures for the wild life, were lack of veterinary care.
\end{abstract}

Keywords: Species of the wild fauna, captivity, handling, conservation, ex situ.

\title{
Introducción
}

Panamá es una de las regiones biológicamente más importantes del planeta, debido a su variada riqueza de ecosistemas y de especies de flora y fauna. Esta variedad se debe a un conjunto de factores, como su historia y su posición geográfica, ubicada en la Región Neotropical como puente natural entre Norte y Suramérica (Valdés, 2008; Farnum Castro, Murillo Godoy \& González, 2020); sin embargo, factores como la deforestación producto de la expansión de la frontera agropecuaria y el creciente desarrollo urbano, la caza furtiva y el tráfico ilegal de animales, han contribuido día a día a su destrucción (Farnum \& Murillo, 2016).

Es por ello, que han surgido mecanismos de protección, conservación, restauración, investigación y manejo de especies, para optimizar las condiciones de estos recursos naturales, promoviendo el uso racional de los mismos, para que cumplan con el marco jurídico de vida silvestre en la República de Panamá. 
Para ello existe el Plan de Estrategias Nacionales para la Conservación de la Diversidad Biológica en países de Centroamérica como: Belice, Guatemala, Honduras, El Salvador, Nicaragua, Costa Rica y Panamá, que plantean la participación de diversos sectores de la sociedad, con el fin de identificar los problemas referentes a la pérdida de la diversidad biológica (Gómez et al., 2010), permitiendo construir lineamientos, bases dentro de este plan estratégico de conservación nacional, entre ellos: la conservación in situ dentro de áreas protegidas, conservación ex situ con tópicos de conservación y uso sostenible, generación y transferencia de tecnología en áreas temáticas de educación ambiental, investigación, entrenamiento, ordenamiento territorial e intercambio de información y distribución equitativa de los beneficios de la conservación (SERNA/GEF/ UNDP/DiBio, 2008); para ejecutar Planes de Acción (SICA/CCAD/CBM, 2003).

Entre las diferentes modalidades de conservación de la fauna silvestre ex situ, están los centros de tenencia y manejo de las especies de vida silvestre que se dividen en centros de fauna son: zoológicos, zoocriaderos, colecciones privadas, serpentarios, acuarios y museos (Consorcio GTZ/FUNDECO/IE, 2001). Estas actividades de conservación se hacen con el fin de preservar muestras de genes y de organismos, para recuperar las poblaciones locales en zonas degradadas, donde la biodiversidad se haya reducido, siempre y cuando no se amenace los ecosistemas, ni las poblaciones silvestres in situ (Valdés, 2008).

La conservación ex situ es una herramienta sumamente importante por tener en cuenta (Baschetto, 2000). La Conservación ex situ de la fauna silvestre en Panamá de acuerdo al Decreto 43, se define como toda actividad de fauna silvestre, que se mantiene en cautiverio, en zoológicos, zoocriaderos, bancos de genes, crio- preservación, colecciones privadas, serpentarios, ranarios, mariposarios e insectarios.

Estos centros deben mantener las condiciones propicias de seguridad necesarias para evitar la entrada o salida de un caso fortuito o de fuerza mayor de los ejemplares; igualmente deberán mantener las condiciones de salubridad, para prevenir la propagación de enfermedades y contar con áreas específicas para exhibición, reproducción y cuarentena, según la actividad (ANAM, 2004).

Dentro de las estrategias de conservación ex situ, la ley 24 de Vida silvestre, establece que la Autoridad Nacional del Ambiente, es la institución encargada de 
promover y regular el establecimiento de zoocriaderos, como una alternativa de aprovechamiento sostenible de la fauna silvestre, disminuyendo de esta manera la caza indiscriminada y la extracción ilegal de algunas especies (ANAM, 2002).

Los ejemplos de domesticación de especies silvestres demuestran claramente el potencial existente de producir animales, alimentos e ingresos (Smythe \& Brown, 1995); pero a nivel individual, un animal debe mantenerse saludable para que pueda sobrevivir, reproducirse y criar su descendencia. La atención individualizada de los animales es especialmente crítica en poblaciones cautivas o silvestres pequeñas. Se hace imperante prestar atención estricta a la nutrición básica del animal, a las necesidades de su ambiente, a los problemas de salud y a los factores de estrés involucrados (Mattiello et al.,1997).

Muchos de estos animales silvestres que residen en santuarios, zoológicos, colecciones privadas y centros de rescate, son ex mascotas de las cuales sus 'dueños' se cansaron, o son animales confiscados por las autoridades luego de ser removidos del cuerpo asesinado de sus madres, producto de la cacería y comercio ilegal. (Khoshen, 2013).

Sin embargo, el seguimiento institucional no ha sido el más adecuado, existiendo deficiencia en la disponibilidad y manejo de información relacionada con: status legal, cantidad de centros de conservación ex situ y de las especies utilizadas, forma de manejo de las especies, especificaciones del tamaño de los recintos adecuados a la especie(s), cumplimiento con las cuotas de liberación del $5 \%$ de los individuos anualmente en el medio natural, de acuerdo a lo establecido en la Resolución AG-0138-2004, que aprueba el manual de procedimientos de ANAM, para acciones sobre la vida silvestre en Panamá.

Es por ello, que el objetivo de esta investigación es actualizar el status, inventariar los sitios y determinar los tipos de centros de cría en cautiverio de especies de fauna silvestres, en el área occidental de Panamá.

\section{Metodología.}

\section{Área de estudio.}


El presente trabajo se realizó en la región occidental de Panamá, en las provincias de Chiriquí, Veraguas, Herrera, Los Santos y Coclé, entre los meses de abril a diciembre de 2014, y de enero a junio de 2015.

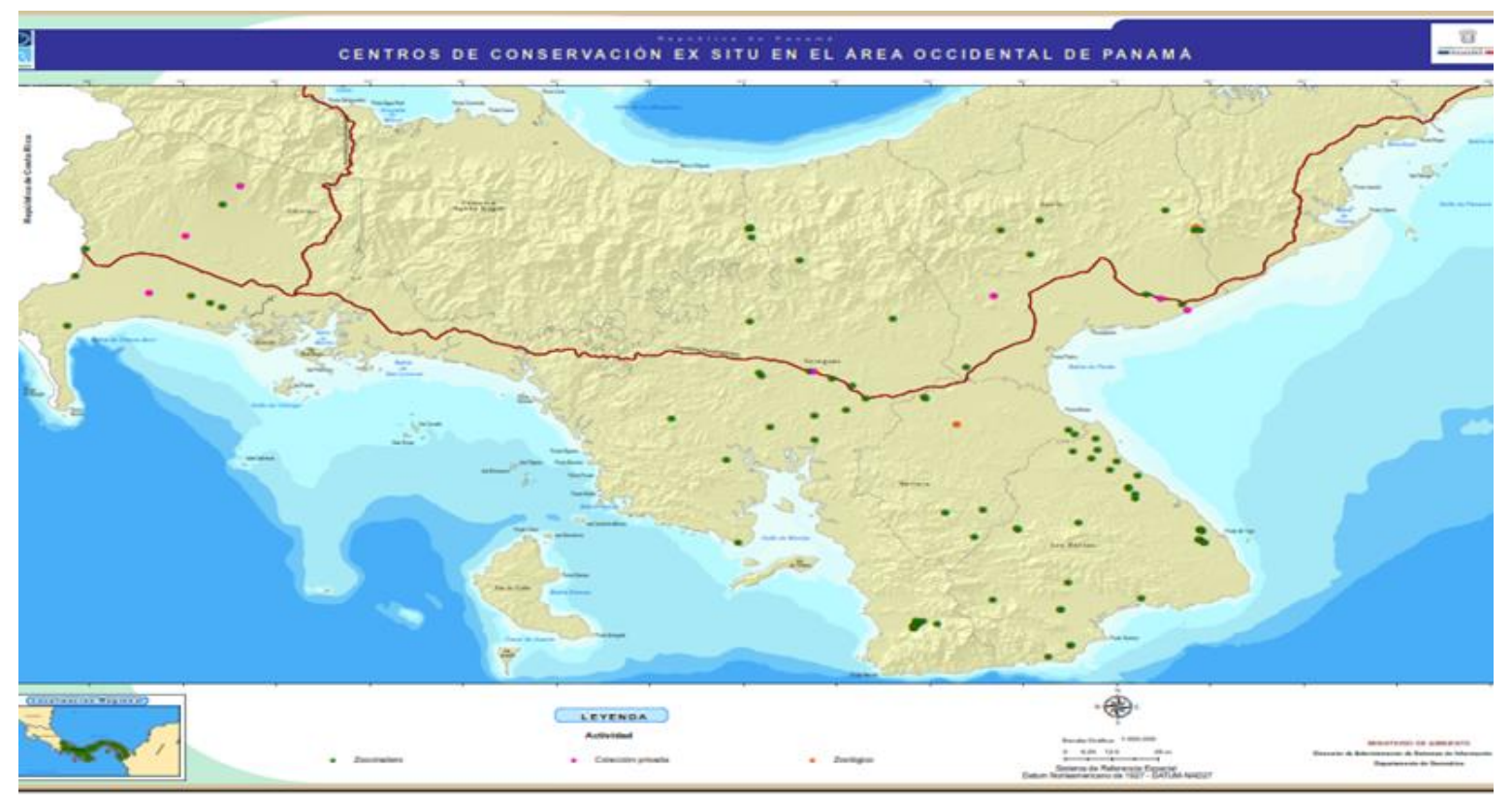

Figura 1. Mapa con la ubicación geográfica de los diferentes sitios de conservación ex situ en la región Occidental de Panamá.

\section{Descripción de la Metodología.}

Para el desarrollo de esta investigación hizo inicialmente la elección del área de trabajo, tomando en consideración la gran incidencia de casos de manejo de fauna silvestre reportados, específicamente en el área occidental de Panamá. Posteriormente, se realizó una revisión de toda la información contenida en expedientes que reposan en el Departamento de Biodiversidad y Vida Silvestre del Ministerio de Ambiente.

Esta información incluyó: informes de actividades, inventarios faunísticos, planes de manejo, actas de entrega de animales y decomisos de fauna, etc. Además, se solicitó información a los funcionarios de vida silvestre de cada una de las Direcciones Regionales del Ministerio de Ambiente.

Una vez colectada toda la información de los registros, antecedentes y consultas pertinentes a las Direcciones Regionales, se procedió a la elaboración de un formulario de inspección, para la toma de datos de los sitios que se visitaron. El formulario de inspección utilizado, contenía la siguiente información solicitada: datos personales de los 
encargados de la actividad, ubicación geográfica (provincia, distrito, corregimiento, lugar o poblado, coordenadas geográficas), tipo de actividad (zoocriadero, colección privada, zoológico), estatus de la actividad (legal, ilegal, trámite, cerrado), inventario de fauna en cautiverio (nombre común, nombre científico, total de individuos), condiciones de los animales (buen estado físico o mal estado), condiciones de los recintos, si existe o no la atención veterinaria en los centros de conservación, tipo de alimentación (semillas, hojas, flores, frutos, tubérculos, alimento preparado u otros); además de documentación fotográfica.

Se organizaron giras de campo en conjunto con el Ministerio de Ambiente, a nivel central y regional, para el reconocimiento y evaluación de los sitios que mantienen actividades ex situ, en cada una de las provincias seleccionadas.

\section{Resultados Y Discusión.}

En la investigación de localizaron en la región occidental de Panamá 111 sitios de conservación ex situ, distribuidos 12 en la provincia de Chiriquí, 44 en la provincia de Veraguas, 8 en la provincia de Herrera, 35 en la provincia de Los Santos y 12 en la provincia de Coclé (Tabla 1, gráfica 1).

Tabla 1. Número de sitios de conservación ex situ, por provincia en la región occidental de Panamá.

\begin{tabular}{|l|c|}
\hline Provincia & Número de sitios \\
\hline Chiriquí & 12 \\
\hline Veraguas & 44 \\
\hline Herrera & 8 \\
\hline Los Santos & 35 \\
\hline Coclé & 12 \\
\hline Total & 111 \\
\hline
\end{tabular}




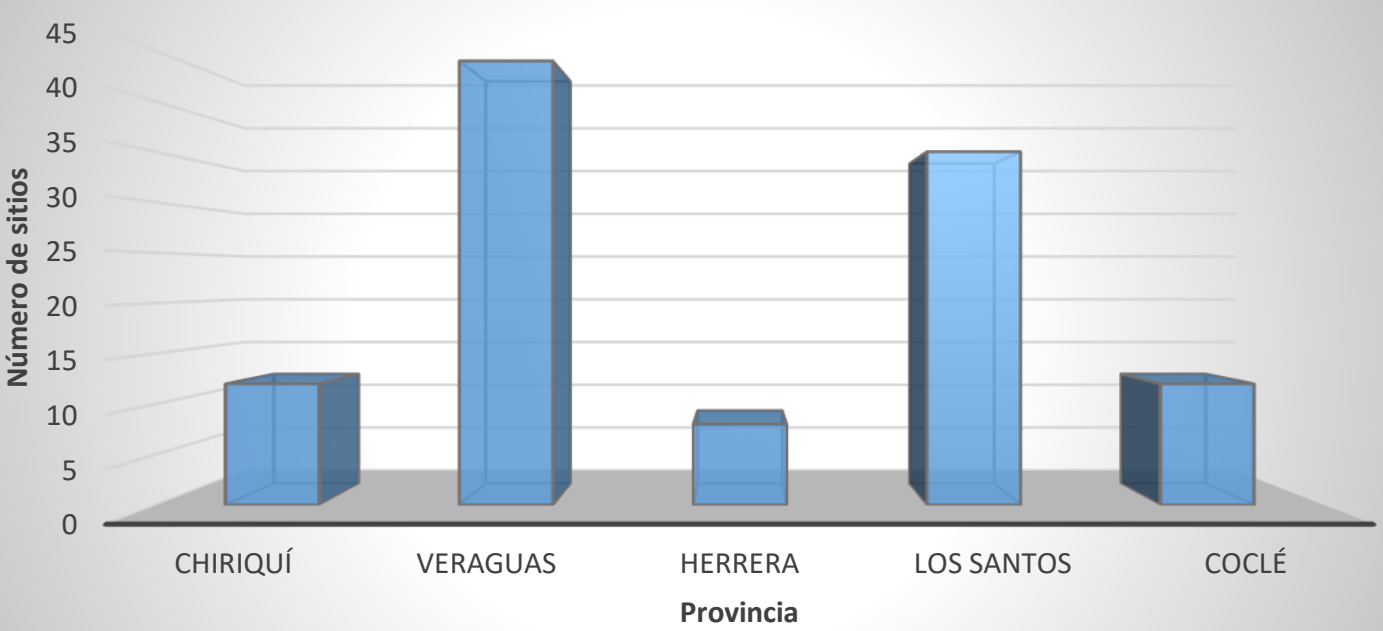

Gráfica 1. Número de sitios de conservación ex situ, por provincia en la región occidental de Panamá.

En la región occidental del país se encontraron tres tipos de actividades de conservación ex situ, tales como zoocrideros, colecciones privadas y zoológicos.

De un total de 102 zoocrideros encontrados en esta región occidental de Panamá, 9 se encontraron en la provincia de Chiriquí, 43 se encontraron en la provincia de Veragua, 7 se encontraron en la provincia de Herrera, 35 se encontraron en la provincia de Los Santos y 8 se encontraron en la provincia de Coclé. También se encontraron 7 colecciones privadas, distribuidas tres en la provincia de Chiriquí, 1 en la provincia de Veraguas y tres en la provincia de Coclé. Además, se encontraron dos zoológicos, ubicados 1 en la provincia de Herrera y 1 en la provincia de Coclé (Cuadro 2, gráfica 2).

Cuadro 2. Actividad y número de sitios de conservación ex situ por provincia, en la región occidental de Panamá.

\begin{tabular}{|l|c|c|c|}
\hline \multirow{2}{*}{ Provincia } & \multicolumn{3}{|c|}{ Actividad de conservación ex situ } \\
\cline { 2 - 4 } & Zoocriadero & $\begin{array}{c}\text { Colección } \\
\text { Privada }\end{array}$ & Zoológico \\
\hline Chiriquí & 9 & 3 & 0 \\
\hline Veraguas & 43 & 1 & 0 \\
\hline Herrera & 7 & 0 & 1 \\
\hline Los Santos & 35 & 0 & 0 \\
\hline Coclé & 8 & 3 & 1 \\
\hline Total & 102 & 7 & 2 \\
\hline
\end{tabular}




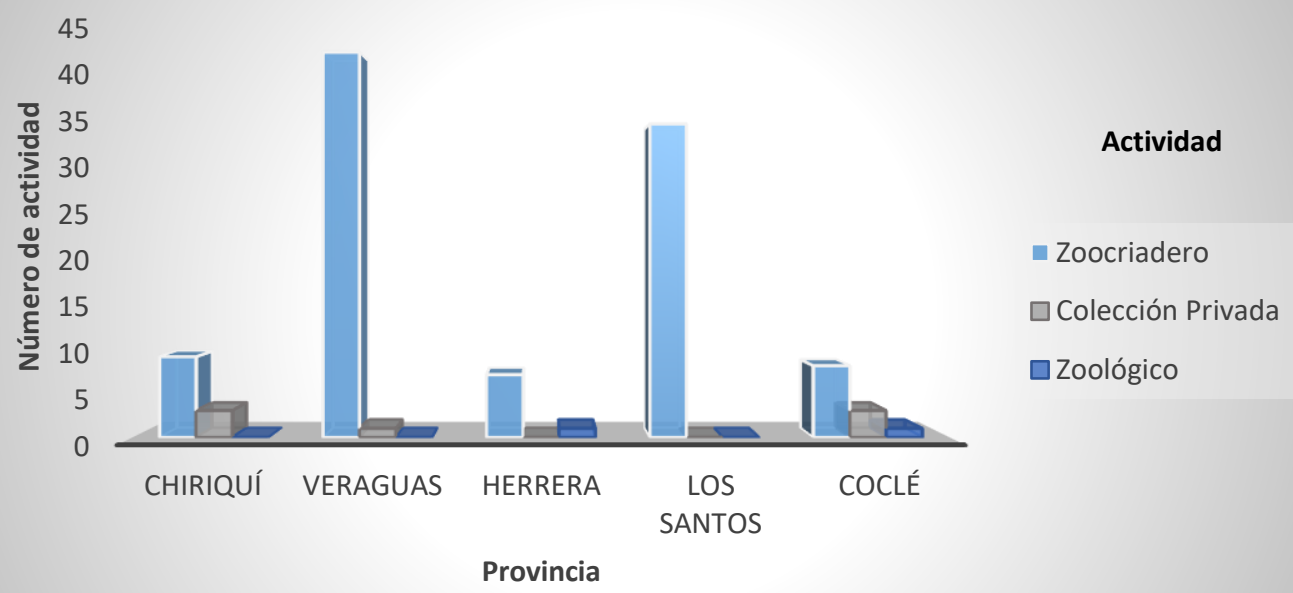

\section{Gráfica 2. Actividad y número de actividades de conservación ex situ por provincia, en la región occidental de Panamá.}

De los 111 sitios con actividad de conservación ex situ en la región occidental de Panamá, se encontró que 38 de estos sitios tenían un trámite legal ante el Ministerio de Ambiente, 35 sitios estaban en trámites y 38 sitios estaban ilegalmente establecidos (Tabla 3, gráfica 3).

Sobre este aspecto del estatus legal de los sitios de manejo y conservación de especies silvestres en cautiverio, la Ley 24 de La Vida Silvestre de 1995, indica que para el establecimiento de los mecanismos de conservación ex situ e in situ, se requerirá de la autorización del INRENARE (hoy Ministerio de Ambiente) a través de la Dirección Nacional de Áreas Protegidas y Vida Silvestre, mediante la expedición de los permisos correspondientes, según los procedimientos administrativos establecidos.

Cuadro 3. Estatus de las actividades ante el Ministerio de Ambiente por provincia, en la región occidental de Panamá.

\begin{tabular}{|l|c|c|c|}
\hline \multirow{2}{*}{ Provincia } & \multicolumn{3}{|c|}{ Estatus } \\
\cline { 2 - 4 } & Legal & Trámite & Ilegal \\
\hline Chiriquí & 1 & 2 & 9 \\
\hline Veraguas & 16 & 22 & 6 \\
\hline Herrera & 4 & 0 & 4 \\
\hline Los Santos & 12 & 5 & 18 \\
\hline Coclé & 5 & 6 & 38 \\
\hline Total & 38 & 35 & 1 \\
\hline
\end{tabular}




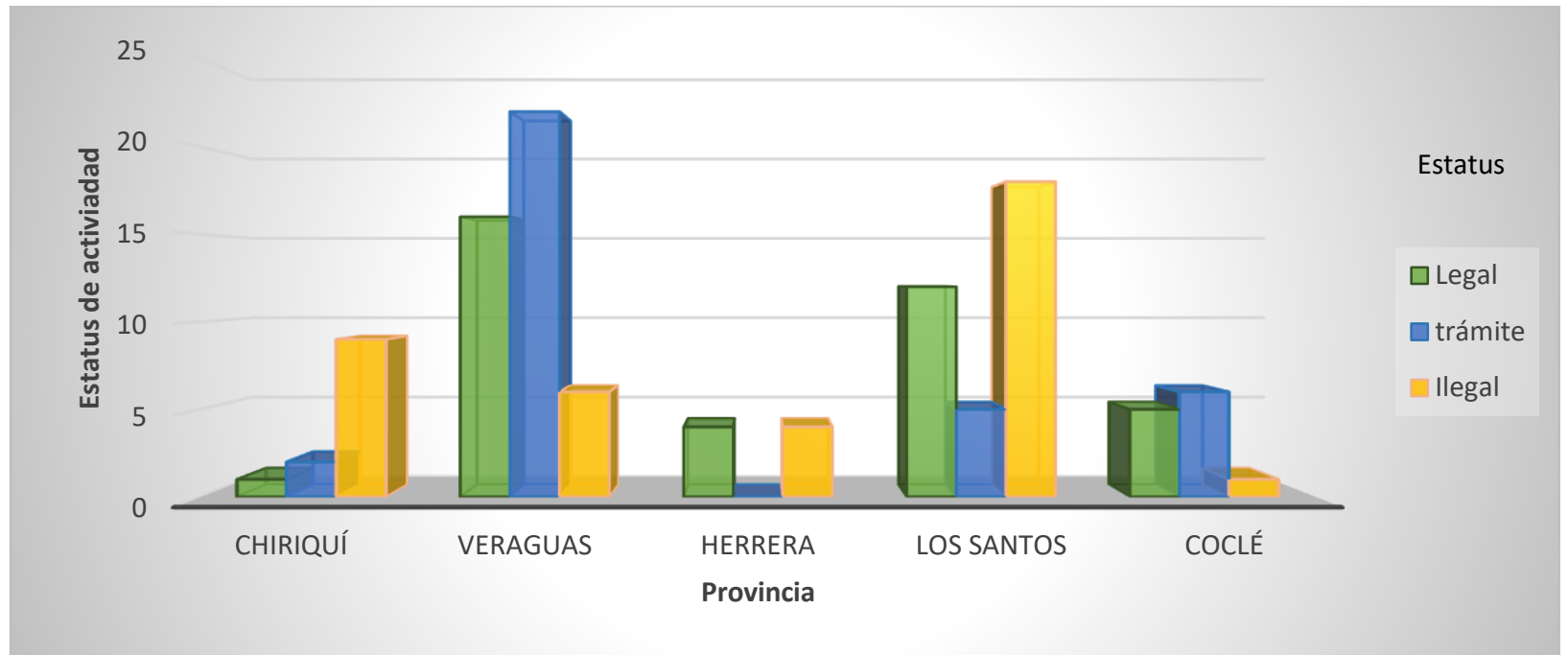

\section{Gráfica 3. Estatus de las actividades ante el Ministerio de Ambiente por provincia, en la región occidental de Panamá.}

De los 111 sitios con actividad de conservación ex situ en la región occidental de Panamá, se encontraron 100 sitios con animales en buen estado físico, 11 con animales en mal estado físico; 109 de las 111 instalaciones eran seguras para albergar a los animales en cautiverio y dos no eran segura; De los 111 sitios con animales en cautiverio encontrados solo 5 contaban con atención veterinaria y 106 carecían de esta atención.

En la provincia de Chiriquí, se encontró que en 11 de los sitios con animales silvestres en cautiverio estaban en buen estado físico y en un sitio los animales estaban en mal estado físico; en la provincia de Veraguas se encontró que 39 sitios que contenían animales silvestres en cautiverio, estos estaban en buen estado físico y en cinco sitios los animales no se encontraban en buen estado físico; en la provincia de Herrera 6 sitios que contenían animales silvestres en cautiverio, estos estaban en buen estado físico, mientras que solo en dos sitos los animales estaban en mal estado físico; en la provincia de Los santos se encontró que 34 de los sitios tenían los animales silvestres en cautiverio en buen estado físico y solo en un sito no se encontraron los animales silvestres en buen estado físico.

En la provincia de Chiriquí y Coclé se encontraron que 11 sitios respectivamente que contenían animales silvestres en cautiverio tenían instalaciones seguras y solo 1 sitio en cada provincia su instalación no era segura; mientras que los 44 sitios de la provincia de Veraguas, los 8 sitos de la provincia de Herrera y los 35 sitios de la provincia de Los Santos, que contenían animales silvestres en cautiverio tenían instalaciones seguras. 
De los 12 sitios con animales silvestre en la provincia de Chiriquí, 2 contaban con atención veterinaria y 10 no contaban con este tipo de atención; En la provincia de Coclé se encontró que tres sitios que contenían animales silvestres en cautiverio, tenían atención veterinaria y 5 sitios no contaban con este servicio; mientras que los 44 sitios presentes en Veraguas, 8 sitos presentes en Herrera y los 35 sitios presentes en Los Santos, que contenían animales silvestres en cautiverio carecían de atención veterinaria (Tabla 4, gráfica 4).

Respecto a las instalaciones seguras, salud de los animales y atención veterinaria para la fauna silvestre en cautiverio ANAM (2004), indica que estos centros deben mantener las condiciones propicias de seguridad necesarias para evitar la entrada 0 salida de un caso fortuito o de fuerza mayor de los ejemplares; igualmente deberán mantener las condiciones de salubridad, para prevenir la propagación de enfermedades y contar con áreas específicas para exhibición, reproducción y cuarentena, según la actividad.

Tabla 4. Número de instalaciones de conservación ex situ con estado físico animal, seguridad del recinto y atención veterinaria por provincia en la región occidental de Panamá.

\begin{tabular}{|l|c|c|c|c|c|c|}
\hline \multirow{2}{*}{ Provincia } & \multicolumn{2}{|c|}{$\begin{array}{c}\text { Estado físico de los } \\
\text { animales }\end{array}$} & \multicolumn{2}{c|}{$\begin{array}{c}\text { Instalaciones } \\
\text { (recintos) }\end{array}$} & \multicolumn{2}{c|}{$\begin{array}{c}\text { Atención } \\
\text { veterinaria }\end{array}$} \\
\cline { 2 - 7 } & $\begin{array}{c}\text { Buen } \\
\text { estado }\end{array}$ & $\begin{array}{c}\text { Mal } \\
\text { estado }\end{array}$ & Seguras & No seguras & $(\mathrm{Si})$ & $(\mathrm{No})$ \\
\hline Chiriquí & 11 & 1 & 11 & 1 & 2 & 10 \\
\hline Veraguas & 39 & 5 & 44 & 0 & 0 & 44 \\
\hline Herrera & 6 & 2 & 8 & 0 & 0 & 8 \\
\hline Los Santos & 34 & 1 & 35 & 0 & 0 & 35 \\
\hline Coclé & 10 & 2 & 11 & 1 & 3 & 9 \\
\hline Total & 100 & 11 & 109 & 2 & 5 & 106 \\
\hline
\end{tabular}




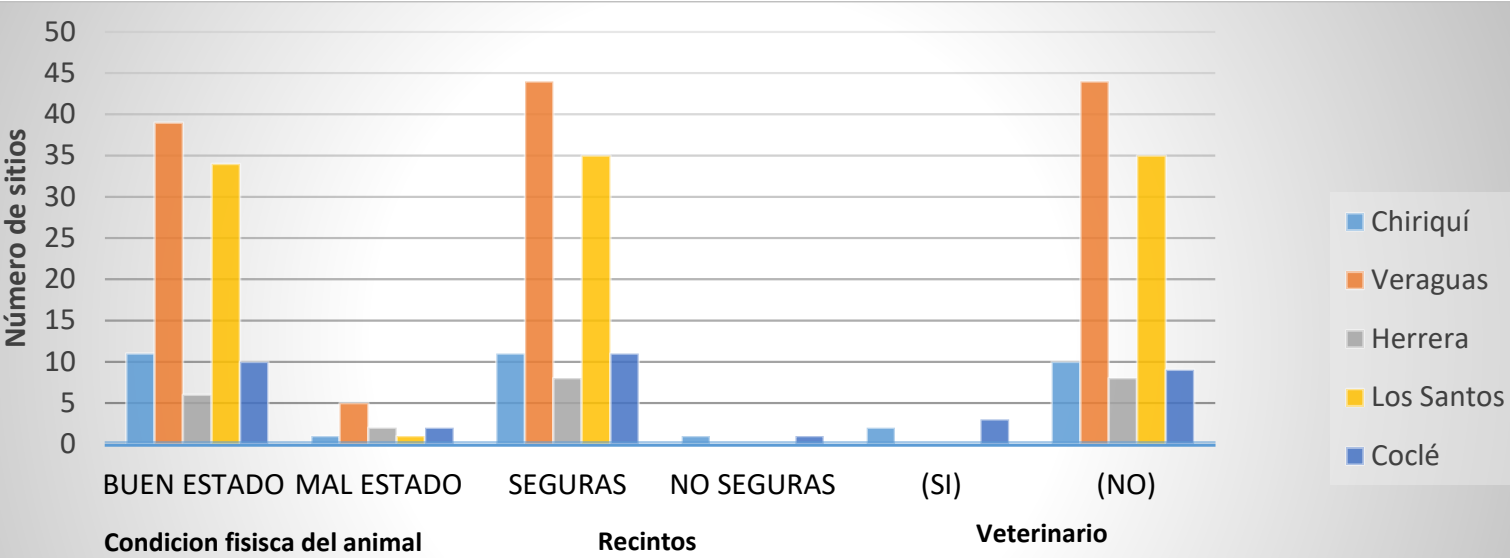

Situación por Provincia

\section{Gráfica 4. Número de instalaciones de conservación exsitu con estado físico animal, seguridad del recinto y atención veterinaria por provincia en la región occidental de Panamá.}

\section{CONCLUSIÓN.}

En la investigación se determinó que en la región occidental de Panamá, existen 111 sitios de conservación de fauna silvestre ex situ, de los cuales 102 fueron zoológicos, 7 fueron colecciones privadas y dos fueron zoológicos, distribuidos en 9 en la provincia de Chiriquí, 43 en la provincia de Veraguas, 7 en la provincia de Herrera, 35 en la provincia de Los Santos y 8 en la provincia de Coclé. De los 111 sitios de conservación de fauna silvestre en cautiverio 38 estaban establecidos legalmente, 38 eran sitios ilegales y 35 estaban en trámite de legalización. En 100 de los 111 sitios de conservación de fauna silvestre ex situ en la región occidental, los animales se encontraban en buen estado de salud física; en109 de los sitios las instalaciones eran seguras para los animales y solo 5 de los 111 sitios de conservación de fauna silvestre exsitu contaban con atención veterinaria. Estos resultados arrojaron la existencia de una gran cantidad de sitios existente en esta región del país, de los que Ministerio de Ambiente desconocía su existencia, su estatus legal, condiciones y atención dada a esa fauna silvestre en cautiverio. 


\section{Referencias Bibliográficas.}

Autoridad Nacional del Ambiente. 1995. Ley 24 del 7 de junio de 1995. "Por la cual se establece la legislación de vida silvestre en la República de Panamá y se dictan otras disposiciones". Vida silvestre de Panamá.

Autoridad Nacional del Ambiente. 2000. Plan de acción nacional sobre la diversidad biológica de Panamá. (Resultados del taller de validación). Programa: Conservación exsitu de la fauna silvestre. ANAM-Panamá.

Autoridad Nacional del Ambiente. 2004. Resolución AG-138-2004 de mayo de 2004. Sobre los procedimientos de vida silvestre.

Autoridad Nacional del Ambiente. 2004. Decreto 43 de 7 de julio de 2004. "Que reglamenta la Ley 24 de 7 de junio de 1995 y dicta otras disposiciones. Vida silvestre de Panamá".

BARRERA, I. 1999. La cría y domesticación del conejo pintado (Agouti paca). Primera edición. Costa Rica-San José. CR.: UICN/ANCON/NORAD. 49 pág.

Baschetto, F. 2000. Repensando los zoológicos de la Argentina. Editorial Dunken. Beldoménico, Antoniazzi, P., Paggi, I. R., Acosta, G., N., Kiener, M., Sguerzo, W., Perassi, R., Camuz, L., Costas, I., Macedo, A., Bravo, C., Acosta, I., Reja, P., Requena, W. 1997. El rol de la medicina veterinaria en la biología de la conservación. Revista Isondú, año II, N.. 3. VEAS.

Consorcio GTZ/FUNDECO/IE. 2001. Conservación ex situ. III Taller regional. Estrategia regional de biodiversidad para los países del trópico Andino.

Farnum Castro, F., Murillo Godoy, V., \& González, F. (2020). Estudio florístico de los fragmentos boscosos de la carretera transístmica (Boyd-Roosevelt) PanamáColón. Universidad Y Sociedad, 12(6), 129-138. Recuperado a partir de https://rus.ucf.edu.cu/index.php/rus/article/view/1823,

Farnum C., F., \& Murillo G., V. (2016). BIODIVERSIDAD Y ASPECTOS ECOLÓGICOS DE LOS PARCHES BOSCOSOS AL BORDE DE LA CARRETERA BOYD ROOSEVELT TRAMO PANAMÁ - COLÓN. Revista Colón Ciencias, Tecnología Y Negocios, 2(2), 49-63. Recuperado a partir de https://www.revistas.up.ac.pa/index.php/revista colon ctn/article/view/1827

GÓMEZ, V., BORDONES, P., MONTAÑO, I., CASAÑAS, O. 2010. Estrategia nacional, para la diversidad biológica de la república bolivariana de Venezuela. Ministerio del Poder Popular de Ambiente. 52. 
Khoshen, H. 2013. Enriquecimiento y bienestar de mamíferos en cautiverioManual para Centro y Sur América. Primera edición. Panamá, República de Panamá. 273 pp. http://enriquecimiento-ambiental.gowildpanama.org/

Mattiello R., Boviez, J., Sánchez A. 1997. "El rol de la medicina veterinaria en la conservación de especies en peligro". Revista Isondú, año II, N. 3. VEAS.

SICA/CCAD/CBM. 2003. Estrategia regional para la conservación y uso Sostenible de la biodiversidad de Mesoamérica. 34 pág.

SERNA, GEF, UNDP, DiBio. 2008. Conservación x situ en Honduras. Evaluación de las capacidades y prioridades de Honduras para la conservación y uso sostenible de la biodiversidad ex situ. Equipo técnico consultor de SERNA, DiBio. 25 pág.

Smythe, N. O. and Brown de Guanti. 1995. Guía de conservación \#26: Domesticación y cría de la paca (Agouti paca). FAO. Roma. 91 pág. http://www.fao.org/docrep/006/v4940s/V4940s01.htm

Valdés, Vanessa. 2008. Prácticas de manejo en la conservación exsitu y su relación con la sostenibilidad ambiental. Tecnología en Marcha, Vol. 21-1, enero-marzo. P. 152-160.

\section{Agradecimiento.}

Nuestro más sincero agradecimiento al Ministerio de Ambiente, en especial a la Dirección de Biodiversidad y Vida Silvestre, por brindarnos su apoyo logístico y colaboración; ya que, sin él no hubiese sido posible la realización de esta investigación.

A todos los Directores Regionales de las provincias de Chiriquí, Veraguas, Herrera, Los Santos y Coclé, que en su momento nos dieron su amistad, tiempo, paciencia y colaboración profesional en cada uno de los sitios visitados durante las giras de campo. 